\title{
HELICAL PULSE LINE STRUCTURES FOR ION ACCELERATION*
}

\author{
R.J. Briggs, SAIC, Alamo, CA, 94507, USA \\ L.L. Reginato and W.L. Waldron, LBNL, Berkeley, CA 94720, U.S.A.
}

\begin{abstract}
The basic concept of the "Pulse Line Ion Accelerator" is presented, where pulse power sources create a ramped traveling wave voltage pulse on a helical pulse line. Ions can surf on this traveling wave and achieve energy gains much larger than the peak applied voltage. Tapered and untapered lines are compared, and a transformer coupling technique for launching the wave is described.
\end{abstract}

\section{INTRODUCTION}

In the "Pulse Line Ion Accelerator" (PLIA) concept a ramped high voltage pulse is applied at the input end of a helical pulse line structure. The resulting traveling wave pulse on the line can accelerate an ion bunch to energies much greater than the peak voltage applied to the line. It should also be possible to achieve an axial acceleration gradient of several $\mathrm{MeV}$ per meter with realistic helix parameters.

The development of this concept was originally motivated by a proposal to use moderate energy intense ion beams to heat matter to regimes of interest for studies of High Energy Density Physics [1]. The concept presented in this paper is an excellent fit to the accelerator requirements for this HEDP application. A helical pulse line inserted into a large bore 5 to 10T superconducting solenoid can transport and accelerate a singly charged Ne or Na ion bunch with a total charge of order 0.1 to 1 micro-coulombs, an axial bunch length of 10's of cm, and a radius of a few $\mathrm{cm}$.

The major attraction of the concept is the very low development and capital cost it promises. The helical pulse line structures are simple to construct and relatively inexpensive. Many features of the wave launching, propagation, dispersion, etc, can be tested on very inexpensive full scale low voltage models. The pulse power drivers are inexpensive (especially at the very low rep rates needed for an HEDP user facility) and only a few are needed to accelerate $\mathrm{Ne}+1$ or $\mathrm{Na}+1$ (for example) to the required $20-25 \mathrm{MeV}$ energies. Finally, the commercialization of high field superconducting solenoids driven by markets in the B $\$$ class, like NMR and MRI, makes them relatively inexpensive compared to the more specialized magnets generally used in particle accelerators.

\section{BASIC CONCEPT}

A sketch of a helical pulse line of radius "a" located inside of a conducting cylinder of radius " $\mathrm{b}$ " is shown in Fig. 1. A dielectric media of permittivity $\varepsilon$ is located in

*Some of this work was performed under the auspices of the U.S. Department of Energy by the University of California, Lawrence Berkeley National Laboratory, Contract DE-AC03-76SF00098 the region outside the helix, while the region inside the helix (where the ion beam is transported) is vacuum. To provide continuous radial focusing of an intense ion bunch, the entire cylindrical structure can be inserted into a large bore solenoid magnet.

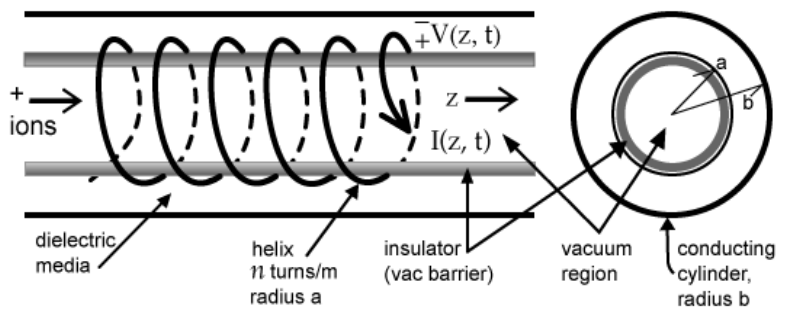

Fig. 1 Sketch of helical pulse line structure

The wave speed regime we are considering for ion acceleration is the order of $1-10 \%$ of the speed of light in vacuum, and the axial wavelength spectrum of interest is large compared to the helix radius. In this regime, the helical line can be modeled as a transmission line with the voltage from the helix to the outer cylinder defined as $V(z, t)$ and the current in the helix as $I(z, t)$, per Fig. 1. The equivalent inductance and capacitance per unit length of the transmission line are $L_{0}=\pi n^{2} a^{2} \mu_{0}\left(1-a^{2} / b^{2}\right)$ and $C_{0}=2 \pi \varepsilon / \ln (b / a)$ respectively, where $\mathrm{n}$ is the number of turns per meter of the helix and the "shielding factor" $\left(1-a^{2} / b^{2}\right)$ in the inductance accounts for the fact that the net axial flux of the sub-microsecond time scale pulsed axial magnetic fields within the conducting cylinder must vanish.

The well known solution to the elementary transmission line equations are traveling waves of arbitrary shape, i.e., for the wave traveling in the $+z$ direction $V(z, t)=V_{+}\left(z-v_{c} t\right)$ with $v_{c}=\left(L_{0} C_{0}\right)^{-1 / 2}$ the circuit wave speed. The current is equal to $V$ divided by the characteristic impedance of the transmission line $Z_{0}=\left(L_{0} / C_{0}\right)^{1 / 2}$. The axial electric field inside the helix is approximately constant in radius in this long wavelength approximation, so the ions are all accelerated by a circuit field given by $E_{z}=-\frac{\partial V}{\partial z}$.

A typical example of the voltage pulse we consider is shown in Fig. 2. At the input end of the helix a pulse power source (using one of the coupling schemes discussed below) creates a ramped voltage waveform in time at a fixed position $\mathrm{z}$ on the helix of the form shown 
in Fig. 2(a). In the ramp region, the voltage goes from $-V_{0}$ to $+V_{0}$ in a timescale $\tau_{c}$.

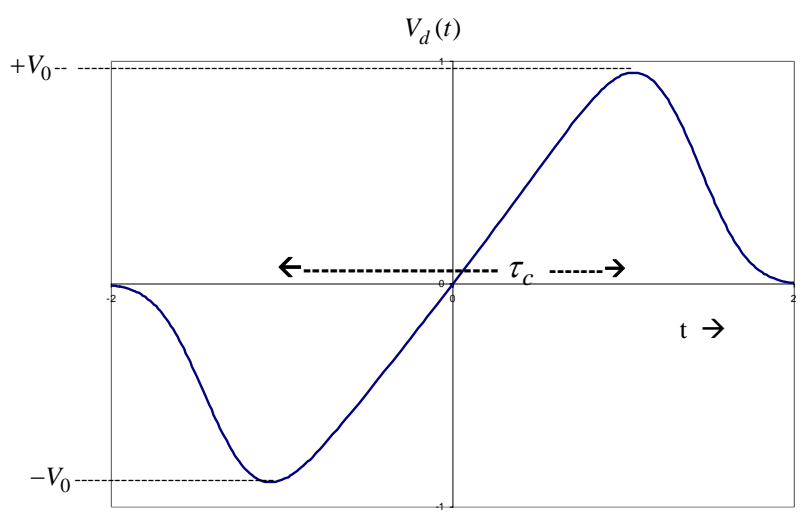

Fig. 2a Drive voltage waveform applied at helix input

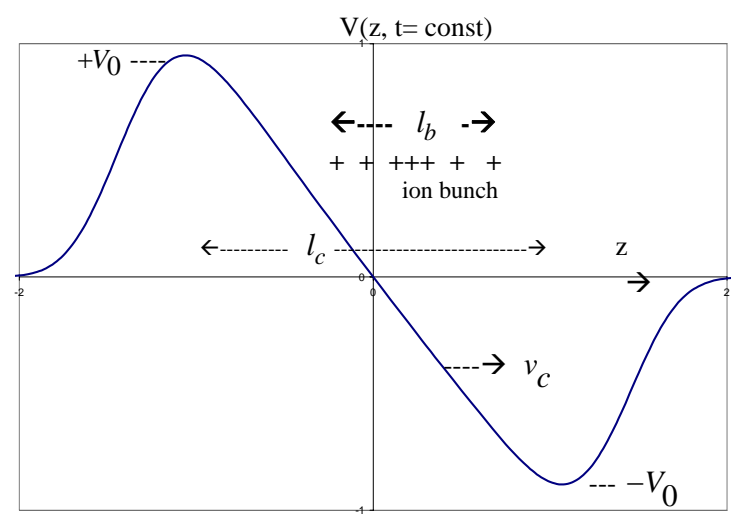

Fig. 2b "Snapshot" of voltage on helix vs. z

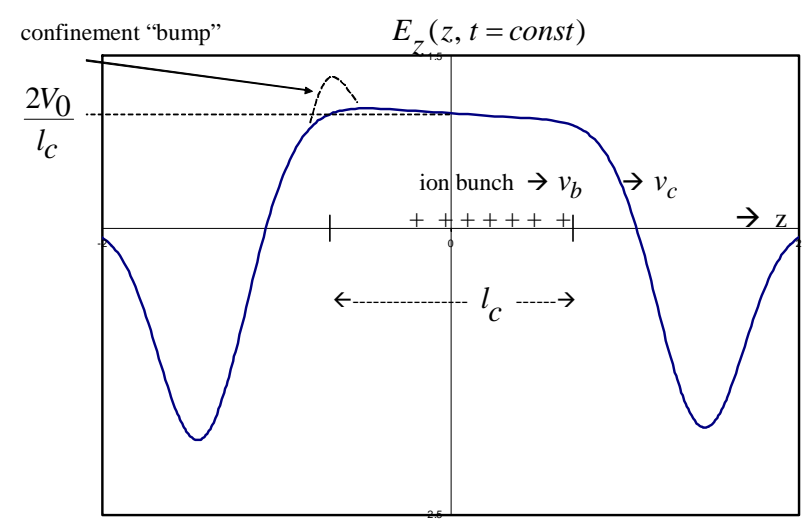

Fig. 2c "Snapshot" of accelerating electric field vs. z

A "snapshot" view of the forward propagating voltage pulse waveform on the helix vs. $\mathrm{z}$ after the wave has been launched is shown in Fig. 2b. The ramp length $l_{c}=v_{c} \tau_{c}$ can be controlled by the input voltage waveform. A "snapshot" of the corresponding accelerating electric field waveform is shown in Fig. 2c. This electric field is approximately constant over the ramp region with an average accelerating gradient of $2 V_{o} / l_{C}$, and a slight negative "tilt" to give axial focusing that compensates for the space charge defocusing field of the ion bunch. An "ear field bump" on the tail of the propagating waveform might also be included to ensure confinement of the ion bunch as it is accelerated.

\section{Gradient Limits}

Factors that will limit the acceleration gradient:

1. The vacuum insulator must hold the pulsed axial electric field without breakdown. We note that the helix automatically provides "inductive grading" of this stress and the typical timescales are a hundred nanoseconds or less. We believe axial gradients on the vacuum insulator of 3 to $5 \mathrm{MV} / \mathrm{m}$ should be reasonably conservative goals for the near term, and more sophisticated insulator designs might substantially increase this vacuum gradient limit.

2 . The peak voltage will be limited by breakdown in the dielectric media $\left(V_{0}<a \ln (b / a) E_{r \max }\right) ; 10-20 \mathrm{MV} / \mathrm{m}$ limits on $E_{r \text { max }}$ should be reasonably conservative for oil or epoxy media with sub microsecond pulses.

3. The ramp length is limited by a requirement for the major fraction of the axial wave number spectra to be confined to the ka<1 region [2]. As a rough criterion, we will take this limit to be $l_{c}<\pi a$.

Limits 2 and 3 combined place a limit on the axial accelerating gradient of $E_{z \max } \sim E_{r \max }(2 \ln (b / a) / \pi)$. With typical design choices of b/a $\sim 1.5$, this limit is also around $3-5 \mathrm{MV} / \mathrm{m}$ for $E_{r \max } \sim 10-20 \mathrm{MV} / \mathrm{m}$.

\section{Acceleration Scenarios}

In a continuous solenoid, the equilibrium beam radius of a space charge dominated beam is independent of particle energy, and proportional to the square root of the line charge density. Acceleration of the ion bunch while keeping its axial length $\left(l_{b}\right)$ and profile constant is therefore a useful "ideal" case to consider.

The acceleration gradient is maximized by choosing the ramp length as short as possible, while still fitting the ion bunch inside the acceleration region (Fig. 2c). One acceleration scenario we consider is a tapered parameter line that maintains $v_{c} \approx v_{b}$ (the ion speed) as the ions accelerate. We pick $l_{c} \approx l_{b}$ at the entrance point and assume that the line is tapered to maintain constant impedance, so the peak voltage $+/-V_{0}$ remains constant. The gradient will weaken down the helix as the ions accelerate and $v_{C}$ increases, since $l_{C} \propto v_{C}$.

An alternative scenario is to use a simple untapered helix. To gain as much energy as possible, the ion bunch should "slide up and down" the potential hill in Fig. 2b as far as possible without having the trailing ions "fall off" the rear part of the accelerating waveform.

A simple calculation of the energy gain with these two scenarios as a function of the helix length (normalized to the ion bunch length) is presented in Fig. 3. The case 
presented assumes an initial ion energy of $10 V_{0}$. We note that the untapered helix performs as well as the tapered helix up to the point where the ion energy increases by $10-15 V_{0}$ (e.g., energy gains of $3-4 \mathrm{MeV}$ in a stage with $V_{0}=300 \mathrm{keV}$ ). Considering the simplicity and flexibility of an untapered line, it will likely be the preferred choice in many, if not most, circumstances.

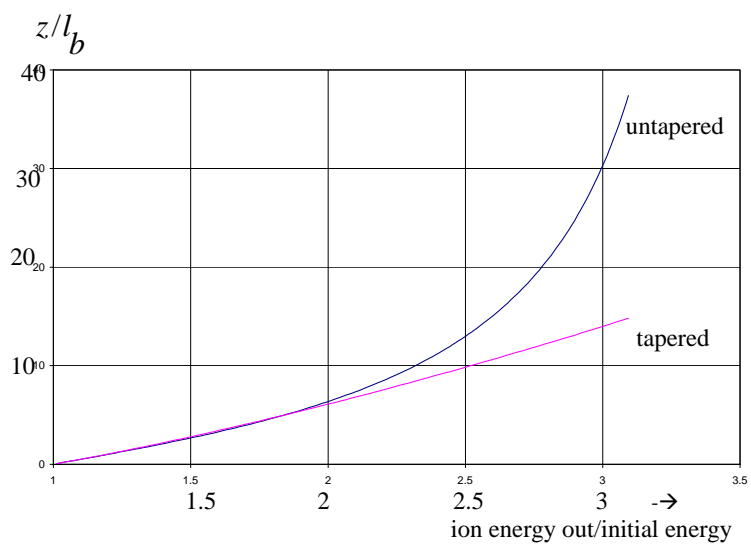

Fig. 3 Comparison of the ion energy gain in untapered and tapered lines for an initial ion energy of $10 \mathrm{~V}_{0}$.

\section{INPUT COUPLERS}

"Direct coupling" of the pulse power source output onto a helix connected to a resistive column, as sketched in Fig. 4, is perhaps the most straightforward approach. Note that as the ion bunch passes through this resistive column region, a head to tail energy variation will generally be induced along the ion pulse by the time varying voltage applied to the helix.

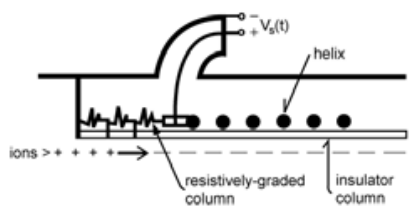

Fig. 4 Direct coupling

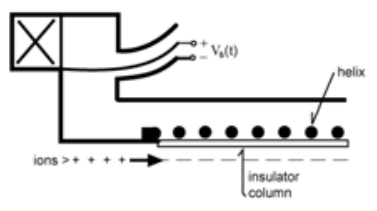

Induction cell coupler
The traveling wave on the helix could also be launched using the induction cell coupler sketched in Fig. 4, where the helix is connected to the beam tube on the left. Using the output voltage of an induction cell to drive the helix eliminates the need for the resistive column and it also eliminates the head to tail energy variation it induces on the ion pulse.

A third method for driving the helix is the transformer coupler shown in Fig. 5. In this approach, the input end of the helix is shorted to the outer cylinder (ground). A primary strap driven by a low impedance pulse power source with (typically) only one or two turns is placed a short distance from the grounded input end of the helix. A fraction of the flux created by the primary strap links the helix, and this flux generates an axial voltage gradient along the input region of the helix. The voltage on the helix at the end of the flux linkage region launches a traveling wave in much the same fashion as if we had connected a direct drive voltage source at that point.
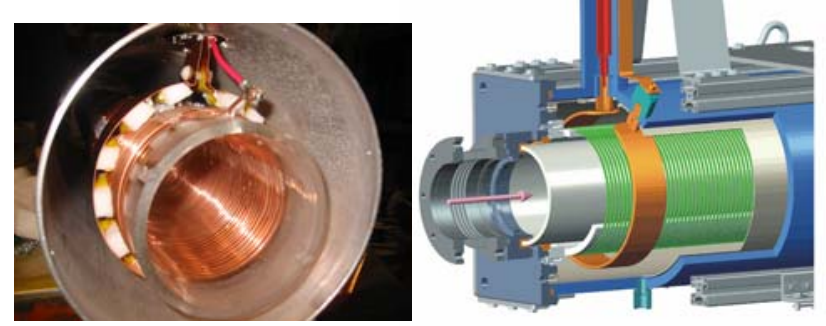

Fig. 5 Single turn primary transformer coupler. The left photo shows the low voltage model used for the first tests of the concept. The cutaway mechanical drawing on the right shows the $8 \mathrm{~cm}$ radius oil helix. [3]

The major advantage of the transformer coupler is the large voltage step-up available. The corresponding reduction in the feed-through voltage is a particularly attractive feature of the transformer coupler approach.

\section{LOW VOLTAGE TESTS}

Tests of the transformer coupling and wave propagation have been made using the air dielectric helix shown in Fig. 5 (6 cm radius helix, $0.9 \mathrm{~m}$ long, $0.25 \mathrm{~cm}$ diameter wires with 2 turns $/ \mathrm{cm}, 10 \mathrm{~cm}$ radius conducting tube, terminated in a $1.5 \mathrm{~K}$ ohm matched load).

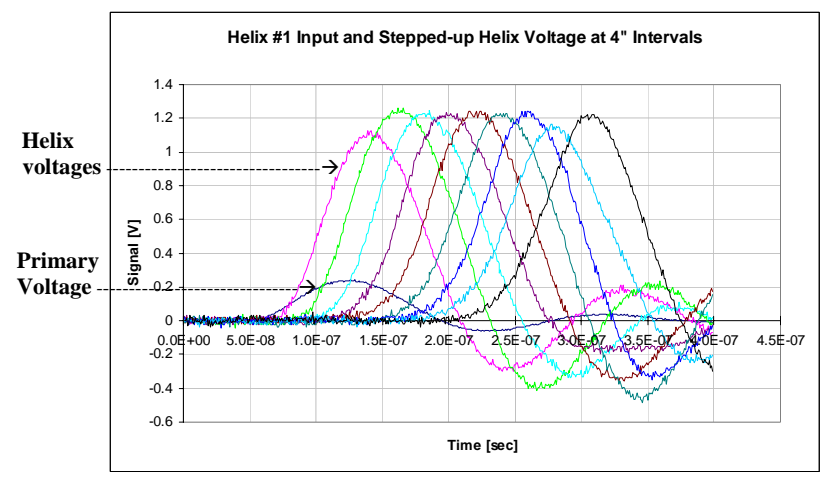

Fig. 6 Pulse voltage waveforms

Measurements of the voltage waveform on the input of the $1 / 2$ " wide primary strap and on the helix at $\mathrm{z}$ locations separated by 4" intervals are shown in Fig. 6. A step up of about 5:1 was demonstrated with this coupler; a step up of almost 10:1 was demonstrated with a 2" wide primary strap. The measured wave propagation speed of $4.6 \mathrm{~m} / \mu \mathrm{sec}$ was in reasonable agreement with the calculated wave speed of $4.8 \mathrm{~m} / \mu \mathrm{sec}$.

\section{REFERENCES}

[1] A. Friedman, et al., "Highly Compressed Ion Beams for High Energy Density Science”, these proceedings.

[2] G. Caporaso, et al., "Dispersion Analysis of the Pulseline Accelerator", these proceedings.

[3] W. Waldron, et al., "High Voltage Operation of Helical Pulseline”, these proceedings. 